\title{
Traumatic caudal elbow luxation in two cats
}

\author{
Paolo Abrescia ${ }^{1}$, Filippo Cinti $^{2 *}$ and Guido Pisani ${ }^{3}$ \\ ${ }^{1}$ Ospedale Santa Fara, Via Generale Nicola Bellomo, 91, 70125 Bari, Italy \\ ${ }^{2}$ Eastcott Veterinary Hospital, Hindle Way, Off Dorcan Way, SN3 3FR, Swindon, Wiltshire, UK \\ ${ }^{3}$ Centro Veterinario Luni Mare, via Togliatti 8/10, 19034 Luni Mare Ortonovo SP, Italy
}

\begin{abstract}
Background: Traumatic caudal elbow luxation is an uncommon injury and it is rarely reported in the cat. Closed reduction is considered in early instance but open reduction and stabilization should be evaluated if the joint cannot be reduced or if gross instability persist.

Case Description: This case report described two Domestic Shorthaired cats referred for monolateral forelimb non-weight bearing lameness caused by trauma. Clinical and radiographic examinations revealed a caudal elbow luxation in both patients. The cats were treated with closed reduction and the elbow joints stabilized at $40^{\circ}$ of flexion by a transarticular external skeletal fixation for 18-22 days. The follow-up examinations at 2 months and at 3 years showed mild and moderate radiographic evidence of osteoarthritis, respectively, but good elbow function in both patients.
\end{abstract}

Conclusion: This technique, for the treatment of the traumatic caudal elbow luxation, is easy and straightforward with few complications and to the authors' knowledge was not previously reported in cats.

Keywords: Closed reduction, Elbow, External fixation, Luxation.

\section{Introduction}

Traumatic elbow joint luxation is a rare condition in dogs and cats (Campbell, 1969; Dassler and Vasseur, 2003; Mitchell, 2011). The considerable stability of the elbow joint is provided by bony conformation, strong collateral ligaments, and the anconeal process that interlocks in the olecranon fossa preventing luxation when the joint is in the extended position (Dassler and Vasseur, 2003; Koch et al., 2005; Griffon, 2012). The most common causes of elbow injury are vehicular trauma or fall from the height (Walker and Hickman, 1958; Campbell, 1969; Dassler and Vasseur, 2003). Frequently direct and indirect forces applied to the elbow typically cause juxta-articular fractures rather than luxation (Walker and Hickman, 1958; Campbell, 1969; Dassler and Vasseur, 2003). When elbow luxation does occur, it is thought to be the result of indirectly applied rotational forces transferred to the elbow via bridging musculotendinous units or ligaments (Campbell, 1971; O'Brien et al., 1992; Farrell et al., 2007). Lateral luxation is more common than medial luxation, this depends on several predisposing factors (Schaeffel et al., 1999). The anatomical conformation of distal humerus showed a large medial epicondyle and the sloped medial condylar ridge (Campbell, 1971; Farrell et al., 2007; Voss and Montavon, 2009; Brinker et al., 2016). In addition, the medial collateral ligament is weaker and has a slim insertion, while the lateral collateral ligament is larger, with a fan-shaped insertion and more elastic due to the presence of the collagen fibers bundles that cross at varying angles (Campbell, 1971; O'Brien et al., 1992). Luxated elbow should be reduced, under general anesthesia, as soon as possible (Campbell, 1971; O'Brien et al., 1992; Sajik et al., 2016). The reduced joint is assessed for collateral stability with Campbell's test maintained the elbow and carpus in a flexed position $\left(90^{\circ}\right)$ and rotating the carpus. If the ligaments are ruptured or avulsed, the paw rotates through a greater range of motion than expected (Campbell, 1971). Open reduction and stabilization should be considered if the joint cannot be reduced or if gross instability is present after closed reduction. Different open techniques are described for treating the elbow luxation, however, there is a better outcome if the joint is stable following closed reduction (Campbell, 1971; O'Brien et al., 1992; Schaeffel et al., 1999; Sajik et al., 2016). Most of the previous literature has focused on canine elbow luxation, with few informations about the feline elbow luxation (Dassler and Vasseur, 2003; Griffon, 2012; Brinker et al., 2016).

In this paper, the authors describe a simple technique to treat the rare caudal luxation of the elbow joint in two cats. To the authors' knowledge, the caudal elbow luxation and closed treatment have not been described previously in the cats.

\section{Case Details}

Two, spayed, Domestic Shorthaired cats with an age of 1.5 years (case 1) and 6 years (case 2) were referred 
to the author's clinic for the non-weight bearing left forelimb lameness after a suspected trauma. Physical examination, of both cats, revealed moderately swollen and painful left elbow, with abnormal posture characterized by extended rotation of antebrachium and supination of the paw. Orthogonal radiographs of forelimbs demonstrated a complete caudal elbow luxation (Fig. 1). The radius and ulna were caudally and proximally displaced in relation to the humerus; furthermore, in case 1, a small avulsion bone fragment (2 $\mathrm{mm}$ diameter) closed to the lateral aspect of the humeral condyle was presented. No other abnormality was identified during the physical and radiographic examinations. The cats were sedated by intramuscular administration of dexmedetomidine (Dexdomitor: Pfizer Italia Srl, Rome, Italy) $(10 \mu \mathrm{g} / \mathrm{kg})$ and methadone (Semfortan: Dechra Veterinary Products Srl, Turin Italy) $(0.4 \mathrm{mg} / \mathrm{kg})$. General anesthesia was induced with propofol (Proposure: Merial Italia Spa, Milan, Italy) $(6 \mathrm{mg} / \mathrm{kg})$ intravenously and, after oro-tracheal intubation, maintained with isoflurane (IsoFlo: Aesica Queenborough Limited, Kent, United Kingdom) and oxygen. Modified closed reduction technique was attempted in both cases. Distal traction of antebrachium with full extension of the elbow was initially achieved followed by simultaneously applying distal pressure on the olecranon and partial elbow flexion. Following successful reduction, the collateral ligaments integrity was indirectly assessed with the Campbell's test (1971), with normal limits value around $110^{\circ}$ of supination and $70^{\circ}$ of pronation recorded in both patients. The joint stability and reduction were maintained with gentle manipulation. However, instability was present at $100^{\circ}$ of elbow extension, while major stability was obtained at $40^{\circ}$ of flexion. For this reason, the elbow was held at $40^{\circ}$ of flexion and a simple transarticular external skeletal fixator type IIa was applied to maintain the stability of the joint. Due to the forced flexion position, which limited the biomechanics movement, the cats were not able to weight bearing in the postoperative

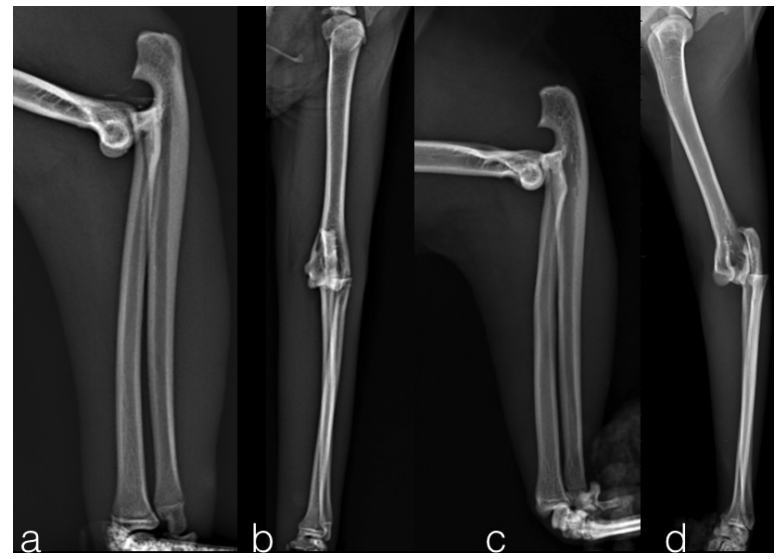

Fig. 1. Pre-operatively radiographs in lateral $(\mathrm{a}, \mathrm{c})$ and craniocaudal (b, d) view. Case $1(\mathrm{a}-\mathrm{b})$; case 2 (c-d). period. Two full pins, smooth Kirschner wires 2.0 $\mathrm{mm}$ of diameter ${ }^{\mathrm{f}}$ were inserted in the proximal third of the diaphysis of the humerus and in the distal third of the radial diaphysis. Stainless steel connecting bars (Gènia. St. Hilaire de Chaléons, France) $(3.0 \mathrm{~mm})$ were positioned medially and laterally connecting with Maynard clamps (Gènia. St. Hilaire de Chaléons, France) to the full pins (Fig. 2). The avulsion bone fragment was not treated in case 1 . The patients were discharged from the clinic 24 hours after surgery. Cage rest without any exercise was recommended for 2 weeks and meloxicam (Meloxoral: Fatro S.p.A., Ozzano Emilia, Italy) $(0.05 \mathrm{mg} / \mathrm{kg})$ was administrated for 7 days.

The case 1 and case 2 were evaluated every 5 days until the final evaluation at 22 and 18 days after surgery, respectively. The implants were well tolerated with acceptable three legs gait in both cats. The owners reported no management's concern of the patient at home. At the final control, the stabilization bars were removed after clinical and radiographic evidence of joint reduction and stability. The Kirschner wires were left for further 5 days later, to be useful in case of reluxation. Protective caps and bandage were applied on the tips of Kirschner wires until their removal. Dermatitis around the pin tracts was the only minor complications associated with the transarticular external skeletal fixation, resolved after implants removal. Thirty days after surgery, in both cases, the clinical examination showed a grade $3 / 4^{\circ}$ lameness (Brunnenberg, 2001), a limited range of motion $\left(150^{\circ} / 70^{\circ}\right)$ and a painless manipulation with no swelling visible. Mild osteoarthrosis was present at radiographic control. For both cats, physiotherapy sessions was not require; however, the owners did some home exercises like gentle passive motions, while restricted movement was continued for another 3 weeks. At 8 weeks after reduction, the cats were no longer lame and the exercise was well tolerated. The range of motion was mildly reduced without pain or joint crepitation. The radiographic follow-up at 2 months after reduction showed mild evidence of osteoarthritis (Fig. 3). The

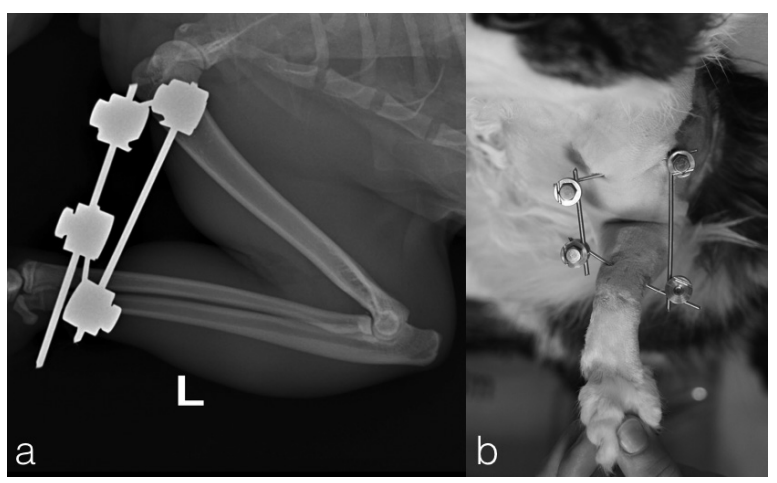

Fig. 2. Transarticular external skeletal fixator type IIa applied to maintain the stability of the joint (case 1). 


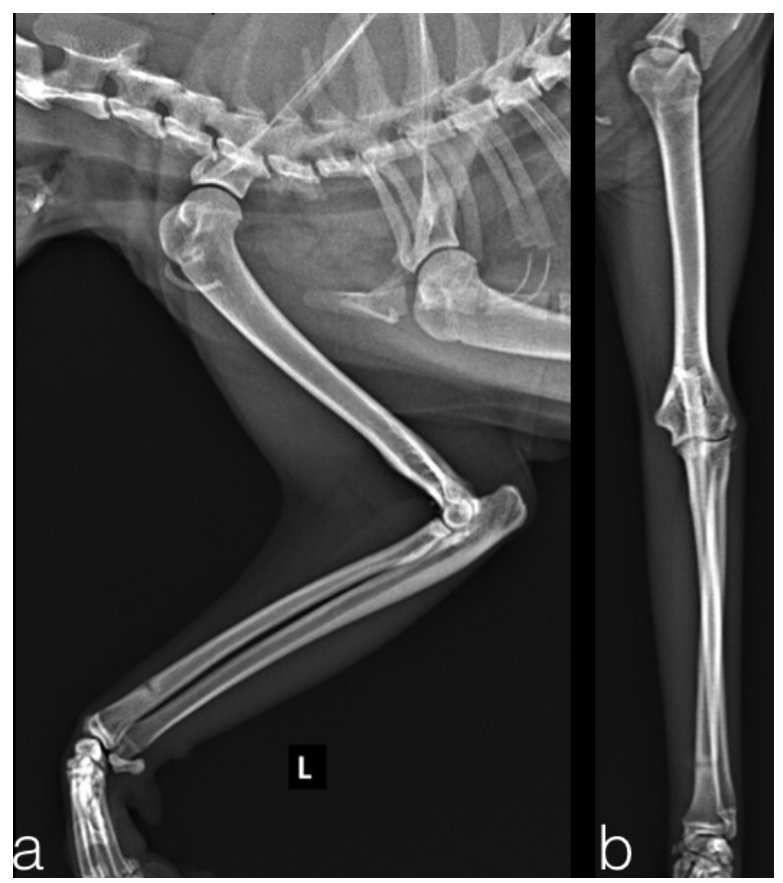

Fig. 3. The radiographic follow-up at two months after reduction showed mild radiographic evidence of osteoarthritis. In the cranio-caudal view (b), near the lateral epicondyle, we can notice a small chip fragment (case 1).

long term follow-up at 3 years assessed with a clinical examination, in both patients, showed normal function and good quality of life despite the mild reduction of the range of motion and moderate development of elbow osteoarthrisis (Fig. 4).

\section{Discussion}

In dogs and cats, more than $90 \%$ of luxation occur laterally (Campbell, 1971; Voss and Montavon, 2009; Sajik et al., 2016); probably due to the conformation of the medial part of the humeral condyle that is larger than its lateral counterpart and its articular surface that has a relatively more acute distal slope (Campbell, 1971). Caudal luxation of the elbow is reported only in one single report of three dogs (Savoldelli et al., 1996) and in one cat treated with open approach, reported by Voss and Montavon (2009) in "Feline orthopedic Surgery and Musculoskeletal disease". To the authors' knowledge, this is the first report described the caudal elbow luxation and close treatment in the cat.

The general appearance of an animal with a caudal elbow luxation is quite simple to recognize. The patient has a $4 / 4^{\circ}$ lameness appearing acutely, the antebrachium and foot are externally rotated, and the elbow is in the extended position. There is usually marked pain with resistance to flexion and extension. Although the basic diagnosis can be made by physical examination, radiographs in two planes are necessary to look for associated fractures and suspect ligaments avulsion (Brinker et al., 2016).

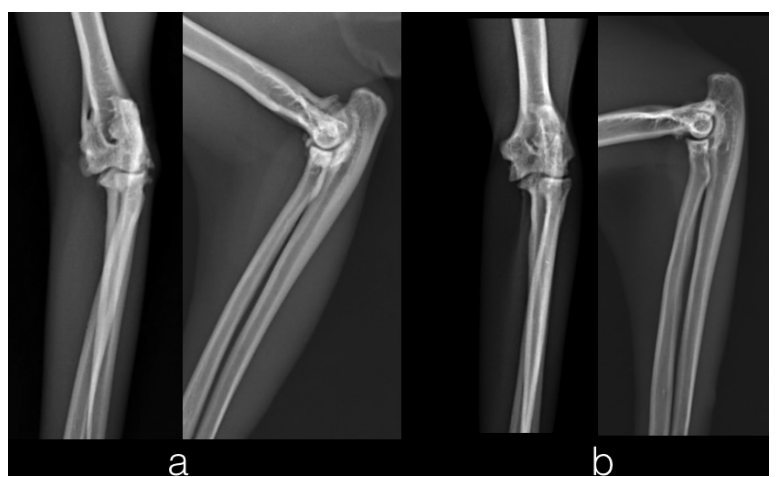

Fig. 4. Long-term radiographic follow-up at three years after surgery showed moderate radiographic evidence of osteoarthritis. Cranio-caudal and medio-lateral views (a-case 1 and b-case 2).

The pathogenesis is unclear; probably the few cases of the caudal luxation of elbow could be explained by rare traumatic events with forces focus applied in the cranial aspect of the radius during the full elbow extension position. In cats, it is very unlikely that such caudal displacement of the radius and ulna could have taken place without severe distension and transection of different local structures, such as the annular ligament, joint capsule, lateral and medial collateral ligaments (Billings et al., 1992; Farrell et al., 2007; Griffon, 2012).

The closed reduction of the luxation should be performed during the first few days after the injury to avoid the muscle contracture that makes the reduction more difficult.

With the animal under general anesthesia, firm palpation was used to establish the position of the humeral condyles relative to the radius and ulna. Due to the rarity of this condition and the absence of instruction in the literature, to reduce the caudal dislocation, we sought a reductive maneuver based on reposition of the bones with additional counteracting distraction forces. In caudal elbow luxation, the proximal dislocation makes the distraction maneuver the first step for reduction; while with the lateral one the repositioning of the olecranon and then extrarotation is the main part of the reduction (Brinker et al., 2016). The small size of the patients and the early treatment make this technique easy with a good final elbow reduction and normal anatomical relationship between the bones. Although most luxations can be reduced in closed manner, in few cases, where closed reduction is not possible or instability persist, open reduction is necessary (Farrell et al., 2007; Griffon, 2012; Brinker et al., 2016). A straight cranio-caudal radiograph of both elbows is necessary to confirm the full reduction. Furthermore, the evaluation of ligamentous damage is crucial after the reduction. Excessive instability, during the Campbell's test (1971), indicates damage to the collateral ligaments, and it is important to decide whether surgical treatment is indicated. If the joint is easily reluxated, surgical repair is necessary. If the 
joint is reasonably stable the decision could be more difficult, and often in these cases, there are a first or second degree of sprains of the ligament which preserves its functional competence. Immobilization will allow healing by fibrosis of periarticular soft tissues and may provide sufficient stability for smaller breeds, especially if they are not working animals (Voss and Montavon, 2009; Brinker et al., 2016). Conversely, surgical treatment is more often indicated in larger and more active animals (Voss and Montavon, 2009; Brinker et al., 2016).

In our cases, the Campbell's test was performed and around $110^{\circ}$ of supination and $70^{\circ}$ of pronation were recorded in both patient. The pronation and supination were within normal limits, and the authors suspected that the collateral ligaments to be grossly intact with only partial tear of the ligament and a mild functional deficit (grade II sprain injury) rather complete rupture (grade III sprain injury) (Voss and Montavon, 2009). Case 1 showed a suspected radiographically avulsion fracture that usually required internal stabilization, nevertheless, the owner declined this surgical treatment for economical reason. The authors decided to perform a closed reduction and stabilization with transarticular external skeletal fixation type IIa. After manual reduction, the instability of elbow was presented at around $100^{\circ}$ of extension, while great stability was maintained with the elbow joint flexed at $40^{\circ}$. To protect against re-luxation, a flexed elbow stabilization was applied that has not previously been described. Despite the unusual technique, it been well tolerated from the patients with a good clinical result. The implants were removed 22 days after surgery for the case 1 and 18 days for the case 2, a longer period in comparison with the one described by Schaeffer et al. (1999), where the immobilization time described should be at 14 days. The delay of implant removal was mainly caused by the absence of literature, for this reason, the authors decided to increase the time of immobilization of 1 week comparing to Schaeffer et al. (1999) indications. However, after the good outcome in both cats, 14 days could be enough to obtain healing and joint stability. In the authors'opinion, external skeletal fixation presents advantages compared to bandages. The advantages of external skeletal fixation are the rigidity, stability, and easy management. On the other hand, the bandage does not require general anesthesia, it is simple to perform, but cannot guarantee rigid stability and require more periodic recheck to reduce the risk of complications. Cats, moreover, usually do not tolerate bandages and in most cases, sedation is necessary for bandage change, for these reasons, the authors considered the external skeletal fixation the best option to avoid stress and bandage complications, like pressure sores on the olecranon (Meeson et al., 2011).

It was decided to use smooth $\mathrm{k}$-wires in order to obtain a simple alignment without a stronger and more expensive construct. According to the authors, a strong construct in the absence of fractures was not necessary to fix the joint for a short period at a predetermined angle. Skin and subcutaneous tissue inflammation around the pin tracts was the only minor complications associated with the transarticular external skeletal fixation, resolved in few days after implants removal. Due to the possibility of re-luxation and the further necessity to restabilize the joint, the Kirschner wires in the humerus and radius were removed 5 days later the bars removal.

Despite the inability of the cats to load on the front limb that was fixed in flexed elbow position, the cats tolerated well the implant. Surely, the treatment with external skeletal fixation creates a sort of disadvantage for life quality, but the short period required for recovery and the speed of return to load justify the choice. The authors hypothesize that the flexible external skeletal fixation could be an options to maintain the elbow reduction and to avoid the impact of immobilization on joint health and range of motion. Postoperative recovery was managed by confining patients to prevent self-trauma and with daily skin medication around the Kirshner wire. Two months after surgery, good clinical outcome and only mild signs of osteoarthritis with marginal osteophytes can be radiographically appreciated, visible in the sagittal view in the lateral compartment. The long-term good clinical outcome, despite the development of elbow osteoarthritis, could be explained by the early treatment, closed reduction technique, the stability of the joint after fixation and the agility and lightness of the feline species. The open surgical approach and ligament reconstruction remain the gold standard in the animal with complete rupture and great elbow joint instability, however, this technique could be an alternative treatment for grade II sprain injuries of the collateral ligaments of the elbow. The unnatural reduction position ( $40^{\circ}$ flexion), like in these cases, maintained for some days didn't resulted any complications (vascular or neurological); however, a decreased range of motion was observed in the post-operative period, possibly due to the trauma and immobilization of the joint for more than 2 weeks.

The functional recovery of the patients did not require physiotherapy sessions; however, the owner was invited to the author to do some home exercises to recover a fluid elbow flexion and extension in the post-operative period.

The small number of cases described and the absence of veterinary literature, especially in cats, make conclusions difficult, but the authors hypothesize that the caudal dislocation of the elbow in the cat can be treated with a closed reduction, temporary flexion stabilization with external skeletal fixation with final good functional recovery.

Further cases are required to determine whether this procedure is optimal for treatment of traumatic caudal elbow luxation in cats and to highlight other complications. 


\section{Conflict of interest}

The authors declared no potential conflicts of interest with respect to the research, authorship, and/ or publication of this article.

\section{Author contributions}

All authors contributed to the conception of the study, study design, acquisition of data, data analysis, and interpretation, as well as drafting and revising and approval of the submitted of the manuscript.

\section{References}

Billings, L.A., Vasseur, P.B., Todoroff, R.J. and Johnson, W. 1992. Clinical results after reduction of traumatic elbow luxation in nine dogs and one cat. J. Am. Anim. Hosp. Assoc. 28(2), 137-142.

Brinker, W.O., Piermattei, D.L. and Flo, G.L. 2016. The elbow joint. In Handbook of small animal orthopedics and fracture repair, 5th ed. Philadelphia, PA: WB Saunders, pp: 325-365.

Brunnenberg, L. 2001. General lameness examination. In Diagnosing lameness in dog, 3rd ed. Oxford, UK: Blackwell Publishing, pp: 102-104.

Campbell, J.R. 1969. Nonfracture injuries to canine elbow. J. Am. Vet. Med. Assoc. 155(5), 735-744.

Campbell, J.R. 1971. Luxation and ligamentous injuries of the elbow in the dog. Vet. Clin. North Am. 1(3), 429-440.

Dassler, C. and Vasseur, P. 2003. Elbow luxation. In Textbook of small animal surgery, 3rd ed. Ed., Slatter D. Philadelphia, PA: Saunders, pp: 19191927.

Farrell, M., Draffan, D., Gemmill, T., Mellor, D. and Carmichael, S. 2007. In vitro validation of a technique for assessment of canine and feline elbow joint collateral ligament integrity and description of a new method for collateral ligament prosthetic replacement. Vet. Surg. 36(6), 548-556.

Griffon, D. 2012. Surgical disease of elbow. In Veterinary surgery small animal, 1st ed. Eds.,
Tobias, K. M. and Johnston, S. A. Philadelphia, PA: Saunders. pp: 724-751.

Koch, R., Hemmes, M., Engelke, E. and Waibl, H. 2005. The collateral ligaments of canine elbow joint: morphometric and structural examination. Kleintierpraxis 50(5), 753-764.

Meeson, R.L., Davidson, C. and Artus, G.I. 2011. Softtissue injuries associated with cast application for distal limb orthopaedic conditions. A retrospective study of sixty dogs and cats. Vet. Comp. Orthop. Traumatol. 24(2), 126-131.

Mitchell, K.E. 2011. Traumatic elbow luxation in 14 dogs and 11 cats. Aust. Vet. J. 89(6), 213-216.

O'Brien, M., Boudrieau, R. and Clark, G.1992. Traumatic Luxation of cubital joint (elbow) in dogs: 44 cases (1978-1988). J. Am. Vet. Med. Assoc. 201(11), 1760-1765.

Sajik, D., Meeson, R.L., Kulendra, N., Jordan, C., James D., Calvo, I., Farrel, M., Kulendra, E. 2016. Multi-ventre retrospective study of long-term outcomes following traumatic elbow luxation in 37 dogs. J. Small Anim. Pract. 57(8), 422-428.

Savoldelli, D., Montavon, P.M. and Suter, D. 1996. Traumatic elbow joint luxation in the dog and cat: perioperative findings. Schweiz Arch Tierheilkd. 138(8), 387-391.

Schaeffel, L., Wolvekamp, P., Meij, B., Theijse, L.F.H., Hazewinkel, H.A.W. 1999. Traumatic luxation of the elbow in 31 dogs. Vet. Comp. Orthoped. Traumatol. 12(1), 33-39.

Voss, K. and Montavon, P.M. 2009. Joint injuries. In Feline orthopedic surgery and muscoloskeletal disease, 1st ed. Eds., Montavon P.M., Voss K. and Langley-Hobbs, S.J. Philadelphia, PA: Saunders Elsevier, pp: 153-160.

Walker, R.G. and Hickman, J. 1958. Injuries to the elbow joint in the dog. Vet. Rec. 70(3), 1191-1194.. 